\section{Minimizing morbidity in radiation oncology: a special issue from Future Oncology}
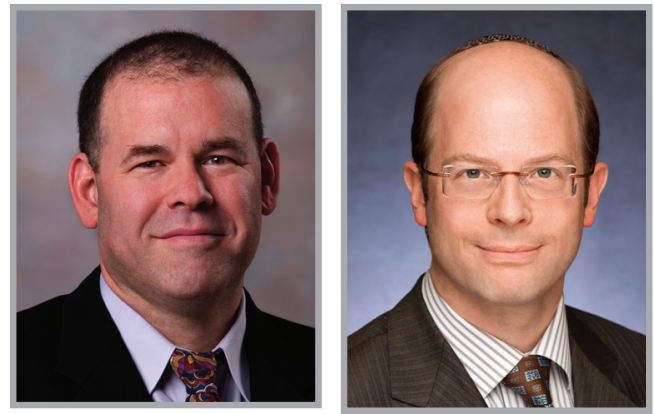

\author{
“Today, more patients than ever who \\ receive radiation therapy experience \\ improved outcomes with lower \\ morbidity than patients treated even a \\ decade ago."
}

Ron Allison ${ }^{*, 1} \&$ Adam Dicker ${ }^{2}$

This issue of Future Oncology highlights some of the accomplishments, limitations and promises of the field of Radiation Oncology, with a focus on means to minimize normal tissue toxicity. Today, more patients than ever who receive radiation therapy experience improved outcomes with lower morbidity than patients treated even a decade ago. This is due in large part to technological advances as outlined in the article by Allison et al. [1]. While the laws of physics that govern radiation therapy has not changed, the ability to accurately deliver tumoricidal doses to the cancer while also reducing dose to critical surrounding tissues has. The widespread use of imaging both during the development and delivery of a patient's treatment has created a new level of treatment accuracy. Various forms of image-guided radiation therapy are currently used, with computed tomography-based most common. As detailed in this article, new forms of imaging including magnetic resonance and biologic approaches may soon come to the forefront, all with the promise of further limiting exposure and dose to normal tissues. Additionally, treatment delivery has simultaneously advanced allowing worldwide introduction of intensitymodulated radiation therapy to become the standard. When appropriate, this delivery technique allows for higher doses of radiation to be delivered to the tumor while simultaneously diminishing radiation dose to normal tissues. Another emerging treatment delivery technique is termed 'radiosurgery.' With these tools, very large doses of radiation are delivered to relatively small volumes. This ideally minimizes normal tissue exposure and injury. With these new technologies may also come unintended consequences. A relatively unusual potential morbidity has been attributed to lung radiosurgery in which rib fracture or injury appears to be enhanced. In the article by Podder $e t$ al., the risk appears to be acceptable and the literature is reviewed [2]. The current state of the art of clinical outcomes for radiosurgery is highlighted by Mahadevan et al.'s report from the 2014 Radiosurgery Society Annual meeting [3]. The future of radiation therapy will also

\section{KEYWORDS}

- radiation morbidity

- radiation oncology

- radiation protectors review

- radiation physics review

- radiation sensitizers review 
include greater reliance on radiation techniques that rely on the Bragg Peak, as found in particle therapy. Here, radiation enters tissue, but does not exit. By accurately placing the Bragg Peak over the tumor, one may avoid clinically significant normal tissue damage. This remains a work in progress.

Despite these rapid technological breakthroughs, a fundamental reality remains. Namely, normal tissues are intertwined with tumor and even with advanced technology these normal tissues are at risk for clinically significant damage during a course of radiation therapy. Today, many tumors cannot be lethally irradiated due to the risk of normal tissue toxicity. Working to enhance the radioprotection of normal tissues and to enhance the radiosensitivity of tumors is a fundamental component of current and future radiation therapy. While several radiosensitizers and radioprotectors are on the market, they are rarely clinically employed. A review of these natural and synthetic compounds is to be found in the articles by both Johnke et al. and Allison [4,5]. Despite nearly 100 years of research, the obstacles to creating successful agents remain high. Still, the need is great. With advances in understanding of the genetic pathways of radiation damage and repair, a new era for these types of radiation modifiers appears to be on our horizon. One such pathway, the renin-angiotensin system appears to influence both blood pressure and response to radiation damage. As described in the article by Hosseinimehr, angiotensin receptor antagonists may enhance radiation treatment efficacy [6]. However, they may also alter normal tissue response including blood pressure. This serves not only as a reminder of the promise of these agents, but also how manipulation of pathways common to both tumors and normal tissues may induce unwanted effects. Closely related is the greater understanding of the immune systems' role both in radiation repair and in damage.

A myriad of immune system pathways are altered by exposure to radiation. As outlined in the article by Agassi et al., while total body exposure appears to downregulate the immune system, focal radiation exposure as is expected during radiation therapy may upregulate select pathways [7]. Indeed, successful treatment may be predicted when immune system components such as $\mathrm{T}$ cells, natural killer cells and memory cells invade the tumor bed following radiation therapy. The review article by Agassi shows the great potential for the combination of radiation therapy and intentional manipulation of the immune process. Perhaps in the near future low doses of radiation, which should not cause permanent damage to normal tissues, may be employed to activate white blood cells, viruses, nanoparticles or other carriers that can ablate tumor. Ideally these techniques would also enhance immune surveillance to prevent tumor spread or recurrence and a vaccine state would be generated.

What may be particularly interesting and relevant to the future of radiation therapy is the apparent wide variation in an individual's normal tissue radiosensitivity. While several inherited diseases are known to make an individual highly sensitive to the effects of radiation, it appears that across the human species normal tissues sensitivity varies widely. As reviewed by Kerns et al., through better understanding of an individual's inherent radiosensitivity, we may be better able to select those individuals at greater risk of morbidity and modify or perhaps avoid radiation therapy [8]. Ideally, individuals at greater risk could assist in the search for radioprotecting agents through enrollment in well-designed clinical trials. While this issue of the journal has focused on minimizing morbidity to those undergoing radiation therapy, the concept of identifying and developing radiation techniques or modifiers that achieve less morbidity has widespread application to any individual exposed to radiation. This includes the large number of individuals undergoing diagnostic radiology, those accidentally exposed to radiation and even potentially for patients undergoing chemotherapy as many of the mechanisms of action appear similar. We are indebted to the journal for devoting space to these important but often underappreciated oncologic concepts.

\section{Financial \& competing interests disclosure}

The authors have no relevant affiliations or financial involvement with any organization or entity with a financial interest in or financial conflict with the subject matter or materials discussed in the manuscript. This includes employment, consultancies, honoraria, stock ownership or options, expert testimony, grants or patents received or pending, or royalties.

No writing assistance was utilized in the production of this manuscript. 


\section{References}

1 Allison RR, Patel RM, McLawhorn RA. Radiation oncology: physics advances to minimize morbidity. Future Oncol. 10(15), 2329-2344 (2014).

2 Podder T, Biswas T, Yao M et al. Chest wall and rib irradiation and toxicities of early stage lung cancer patients treated with CyberKnife stereotactic body radiotherapy. Future Oncol. 10 (15), 2311-2317 (2014).

3 Mahadevan A, Bucholz R, Gaya A et al. Best of the Radiosurgery Society ${ }^{\circledR}$ Scientific
Meeting 2014: stereotactic radiosurgery/ stereotactic body radiotherapy treatment of extracranial and intracranial lesions. Future Oncol. 10(15), 2307-2310 (2014).

4 Johnke RM, Sattler JA, Allison RR. Radioprotective agents for radiation therapy: future trends. Future Oncol. 10(15), 2345-2357 (2014).

5 Allison RR. Radiobiological modifiers in clinical radiation oncology: current reality and future potential. Future Oncol. 10(15), 2359-2379 (2014).
6 Hosseinimehr SJ. The use of angiotension II receptor antagonists to increase the efficacy of radiotherapy in cancer treatment. Future Oncol. 10(15), 2381-2390 (2014).

7 Agassi AM, Myslicki FA, Shulman JM et al. The promise of combining radiation therapy and immunotherapy: morbidity and toxicity. Future Oncol. 10(15), 2319-2328 (2014).

8 Kerns SL, West CML, Andreassen CN et al. Progress in radiogenomics, Future Oncol. 10 (15), 2391-2406 (2014). 